\title{
Multimedia Big Data Frame Combination Storage Strategy Based on Virtual Space Distortion
}

\author{
https://doi.org/10.3991/ijoe.v13i02.6611 \\ Jian Luo \\ Zhejiang Technical Institute of Economics, Hangzhou Zhejiang, China \\ jiansluo@yahoo.com
}

\begin{abstract}
Aiming at the transmission reliability and storage management of multimedia data, the multimedia big data frame combination storage strategy is proposed based on the virtual space distortion analysis model. On the one hand, the virtual space distortion analysis model is established. This model can be used to find the high similarity of video frames and flow structures in different multimedia streams. The model can be used to uniform the multimedia stream state and effectively improve the efficiency of data mining. The model can effectively analyze the difference between the multimedia stream of virtual space and the storage of the server group. On the other hand, based on the distorted efforts of the virtual space and heterogeneous of the multimedia data stream, combined with big data multimedia service requirements, the big data frame combination of storage space and virtual space integration would be integrated as a whole through the multimedia data frame combination and distorted perception. Finally, the combination of multimedia data frames is proposed. The delay, channel, storage and big data processing level of the virtual space storage scheme and were analyzed and evaluated by the simulation experiment, compared with the physical storage scheme of the multimedia big data. The experimental results show that the proposed scheme has significant advantages in the above aspects.
\end{abstract}

Keywords-Multimedia stream, big data storage, frame combination, virtual space, space distortion

\section{$1 \quad$ Introduction}

Big data processing, transmission and storage has gradually become an important development direction of multimedia communication [1] [2]. It brings many challenges to realize the real time transmission [3] and the high efficiency storage [4]. In the era of multimedia data, the structure of the multimedia data is normalized. It is the key problem how to realize the real-time processing and efficient storage of big data [5].

In the research of virtual space and environment, the article [6] investigated the basic satellite-terrestrial architecture of space information networking, and presented the virtual multi-beamforming scheme for distributed satellite clusters. The article [7] 
proposed the virtual space vector modulation technique for reducing both magnitude and third-order harmonic component of the common-mode voltage in a two-level voltage-source inverter.

About the study of multimedia transmission, the authors proposed a cooperative multimedia transmission protocol based on a novel merge-and-forward relaying and the best relay selection schemes [8]. The high-complexity classical robust solution distribution scheme was proposed in article [9] by low constant average degree distributions with high intermediate symbol recovery rates. In article [10], the scheduling of multimedia transmissions over drive-thru Internet was investigated to support multimedia services for people on the road.

About big data processing and storage, the big data capabilities was applied to semiconductor manufacturing advanced process control, which can finer levels of control and diagnostics [11]. The authors of article [12] derived the incremental programs that return accurate results by retaining a minimal state during the query evaluation lifetime and using a novel incremental evaluation technique. The authors used the information from movie ratings, which could result in a movie recommendation in implementing cosine similarity.

The research results of the above research on the relationship between the virtual environment and the multimedia stream are not deeply studied. The impact of big data storage on multimedia communication quality and the need for efficient storage solutions should be further studied. The rest of the paper is organized as follows. Section 2 describes the virtual spatial distortion analysis model. The multimedia big data frame combination storage is studied in Section 3. The performance analysis of big data storage mechanism was completed in Section 4. Finally, the conclusion of this paper was given in Section 5.

\section{Virtual Spatial Distortion Analysis Model}

In the structure of multimedia server cluster, multimedia streaming is classified and managed by the storage architecture of cluster head server. The load of virtual multimedia data space would be mined according to the hierarchical model of multimedia streaming. The mining task between the virtual multimedia data stream and the server includes the frame structure characteristic of the multimedia data and the real-time response delay of the server data mining. Here, the multimedia stream state of the virtual space is updated according to the mining results of the server. Virtual space server data mining should complete the flow structure update, the redundancy between the frame measurements, the available physical storage space of the servers and other tasks. There is a linear relationship between the multimedia stream of virtual space and the scale of the multimedia data stored by the server. The more the difference of multimedia streaming in virtual space, the higher the data mining complexity of multimedia server group.

Find the video frame high similarity and flow structure in different multimedia streams, to reduce the difference of data mining multimedia virtual space complexity and the weakening of the state consistency processing and data flow inside the comm- 
Short Paper-Multimedia Big Data Frame Combination Storage Strategy Based on Virtual Space Distortion

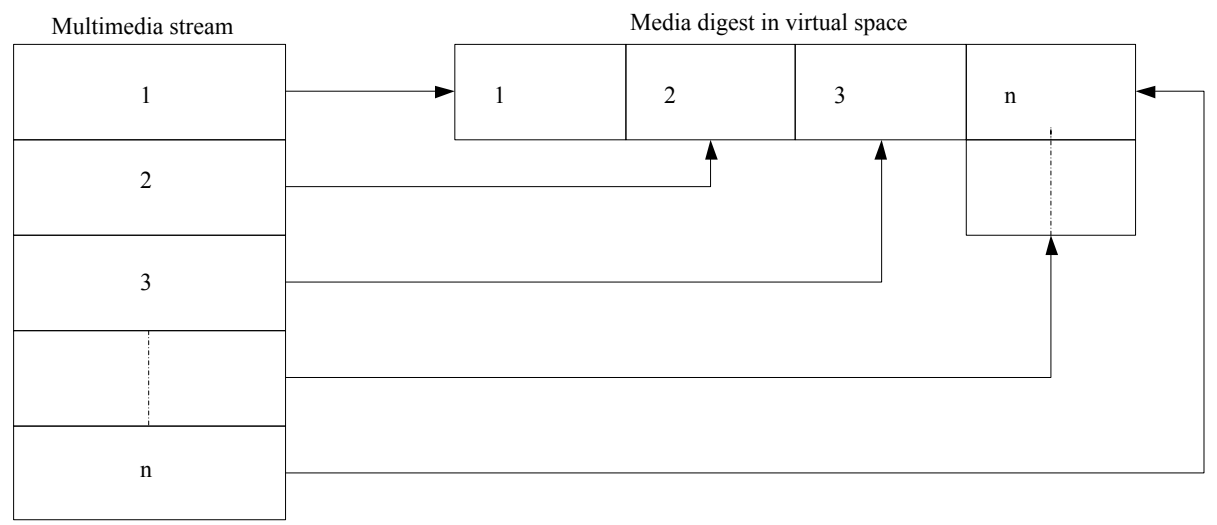

Fig. 1. Virtual digest space for multimedia streaming

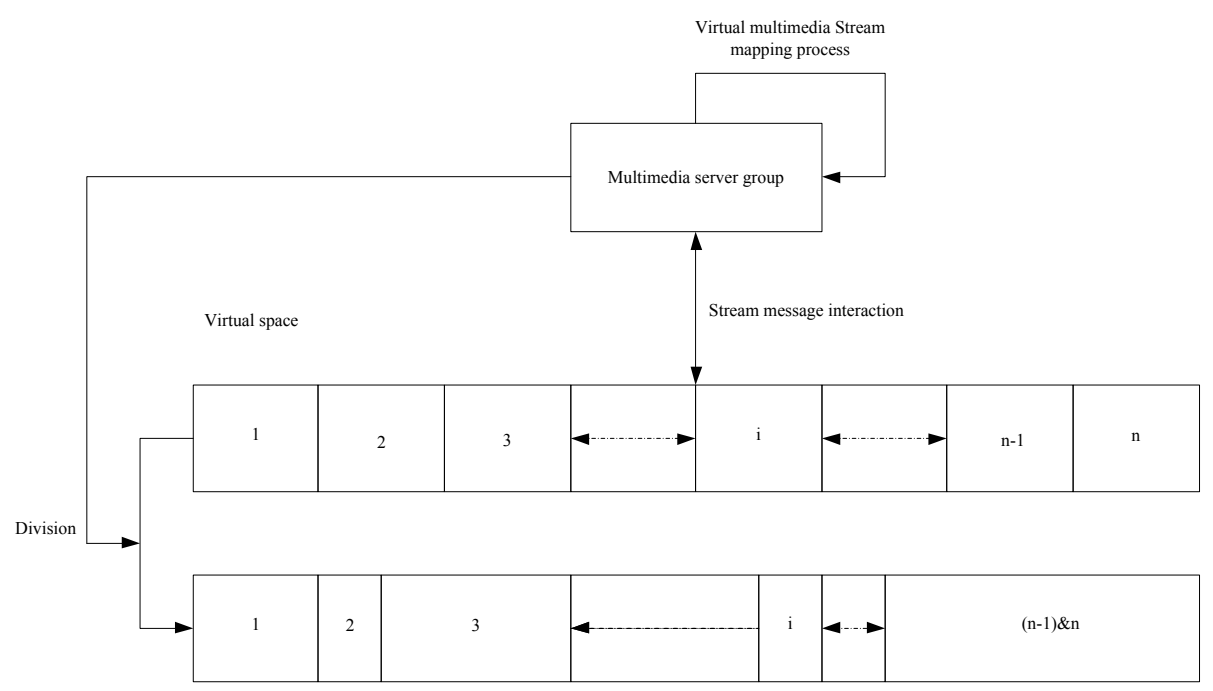

Virtual space after division

Fig. 2. Segmentation of virtual space

unication server group. The multimedia data mining results of the server can map the hot spots of the multimedia stream in the virtual space. Therefore, to set a summary for each multimedia stream, as shown in Figure 1. Within a server cluster, a plurality of servers can carry out multimedia computing and data mining through the summary, and write the results to the abstract, and then interact with other servers to virtual space. Conflict differentiated multimedia stream when virtual space, virtual space server based on message flow, by increasing the delay of network news broadcast, multimedia streaming server re segmentation map, as shown in Figure 2. 
The multimedia stream of virtual space and the corresponding server establish the mapping relationship based on the abstract. During the operation of the server group, the change of the scale and the structure of the multimedia data, the change of the storage mode and the space utilization rate of the server. Then through the virtual space for multimedia streaming updates and message flow interaction, multimedia streaming and storage management will be converted to a physical server response and reconstruction, effective division of multimedia data to realize the virtual space.

Assuming the server group's independent server data mining cost $\mathrm{C}_{\mathrm{S}}$, the server streaming message broadcast $\operatorname{cost} \mathrm{C}_{\mathrm{C}}$. If the number of the virtual space of the multimedia stream is $\mathrm{m}$, the number of the server is $\mathrm{k}$, the virtual load of any two multimedia stream $\mathrm{i}$ and $\mathrm{MS}_{\mathrm{i}}$ is $\mathrm{j}$ and $\mathrm{MS}_{\mathrm{j}}$, then the data mining work load of the server group is shown in the formula 1 . The management load of these 2 multimedia streams is shown in formula 2.

$$
\begin{array}{r}
L_{S G}(k)=\sum_{i=1}^{k} C_{C}\left(C_{S}-\frac{M S_{i}}{M S_{i-1}}\right) \\
M_{S(i, j)}=\frac{m}{n(i+j)} \sum_{p=1}^{|i-j|} \frac{M S_{j}}{M S_{i}} L_{S G}(|i-j|)
\end{array}
$$

Due to the re segmentation in the virtual space and server in the process of restructuring, the frame structure changes, changes in the structure of server storage system workload and the increase of multimedia virtual space abstract updates and other factors, so that the server group storage error. This error creates distortions in the virtual space. In particular, the error of the multimedia data in the larger and the storage space is larger. These large error data is a waste of storage space. These errors have brought enormous computational burden to the virtual space. Therefore, it is necessary to analyze and model the twisted space.

Summary of statistical multimedia streaming data distortion samples. Based on these distorted samples, the distorted area of the virtual space is calculated by AT and the arc RT, as shown in the formula 3. These two parameters determine the credibility of the virtual space which is connected with the distorted part. The reliability directly affects the storage architecture and data mining complexity of other concurrent multimedia streams.

$$
\left\{\begin{array}{l}
A_{T}=\alpha \sum_{i=1}^{k}\left|M S_{i}-M S_{(i, i+1)}\right|^{2} \\
R_{T}=\sum_{i=1}^{k}\left|\operatorname{angle}\left(O_{i, i+1}, O_{i-1, i}\right)\right|
\end{array}\right.
$$

Among them, $\alpha$ is the intensity of distortion. Function angle is used to solve the distortion of the sample and the angle of distortion of the original sample.

The statistical parameters of the virtual space to distortion, loss of multimedia virtual space is converted to the true distortion, the distortion by formula 4 is optimized to reduce the impact of multimedia data storage servers and virtual space optimization model.

The statistical parameters of the virtual space to distortion, loss of multimedia virtual space is converted to the true distortion, the distortion by formula 4 is 
optimized to reduce the impact of multimedia data storage servers and virtual space optimization model.

$$
\left\{\begin{array}{l}
\overline{A_{T}}=\alpha\left|\sum_{j=1}^{k-q}\right| M S_{i}-\left.M S_{(i, i+1)}\right|^{2}-\sum_{i=1}^{q}\left|d j_{i}-d j_{(i, i+1)}\right|^{2} \mid \\
\overline{R_{T}}=\frac{\sum_{i=1}^{k}\left|\operatorname{angle}\left(O_{i, i+1}, O_{i-1, i}\right)\right|}{\sum_{i=1}^{q} \sum_{u=1}^{k-q} \operatorname{angle}\left(d j_{u}, d j_{u-i}\right)}
\end{array}\right.
$$

Among them, parameter $\mathrm{q}$ represents the number of distorted samples. Distorted area after optimization for virtual space. The sample distortion after the optimization of virtual space is distorted. In comparison with the formula 3 , it is found that the 2 parameters are significantly reduced.

\section{Multimedia Large Data Frame Combined Storage}

Multimedia big data service based on the heterogeneous data and distorted virtual space, to provide a description model for multimedia services. The model includes the basic information of multimedia, the relationship between large data structure and storage requirements. Design service interface and distortion sensing interface between virtual space and server of multimedia large data. In the entity big data multimedia service, the multimedia service takes the stream communication as the core. The service pays attention to the dynamic characteristics of the service. However, for the large data services based on virtual space, the characteristics of large data and multimedia data storage is the core of the virtual space. Virtual space based multimedia data service requires large data service interface to perceive the virtual space distortion and update the storage characteristics of large data.

For large data multimedia services based on virtual space, multimedia services according to the data frame combination expression by distorting the logical drive, large data frame combination of storage space and virtual space integration as a whole. Based on the characteristics of multimedia services and large data structure, the combination of the large data frame is designed by the collaborative cooperation of the virtual space distortion module. The storage planning through the virtual space of different multimedia services between the linear logic of the development of large data frame combination of data sequence input and output matching relationship matrix.

Multimedia large data frame combined storage model provides the realization of the mapping of large data frame combination of virtual space and large data services. For the virtual space distortion driven large data storage, we need to meet the following conditions:

1. to establish a distorted perception model and interface. They are used to provide heterogeneous data source transparency and diversity of multimedia services for multimedia data storage services. 
2. the relationship between the effective capture of the large data frame of the multimedia data frame and the distortion of the virtual sub space. The efficiency and complexity of real time statistics of large data storage. The above results can effectively support the cross platform characteristics of multimedia data services and multimedia service quality under the distorted environment of large data services.

The combination of large data frame can describe the structure of multimedia streaming, the mapping of virtual space and the ability of server storage. A large data frame can be described as $<\mathrm{MC}$, VSM, SA, TW $>$. Among them, MC represents the expression of the multimedia stream structure in virtual space. VSM representation of the virtual space of the multimedia large data mapping matrix. SA represents the server storage capacity of virtual space. TW the distortion of the virtual space of the virtual space. The large data frame of the multimedia stream has the following relations:

1. The XOR operation results of $1 \mathrm{MC}_{\mathrm{i}}$ and $\mathrm{MC}_{\mathrm{j}} \mathrm{i}+\mathrm{j}$ expression as a binary representation of the frame.

2. $\mathrm{VSM}_{\mathrm{i}}$ is a subset of VSM.

3. SA according to the transmission efficiency and broadcast quality of heterogeneous multimedia streaming.

4. $\mathrm{TW}_{\mathrm{i}}$ and $\mathrm{TW}_{\mathrm{j}}$ and the need to go through the virtual space of the distorted interface matching.

5. $<\mathrm{MC}_{\mathrm{i}}, \mathrm{VSM}_{\mathrm{i}}, \mathrm{SA}_{\mathrm{i}}, \mathrm{TW}_{\mathrm{i}}>$ and $<\mathrm{MC}_{\mathrm{j}}, \mathrm{VSM}_{\mathrm{j}}, \mathrm{SA}_{\mathrm{j}}, \mathrm{TW}_{\mathrm{j}}>$ encoding constraints are always consistent.

Among them, $i$ and $j$, respectively, which represent the big data frame of the multimedia stream. Big data frame combination and inter frame relations are shown in Figure 3.

The distortion mapping between the multimedia big data frame combination and the virtual space is between the multimedia service interface and the distorted sensing interface. Combining the logical relationship between the multimedia data frame sequence and expression characteristics of large data, multimedia data frames for multimedia storage specification mapping large data streams and large data structure, the virtual space characteristics, multimedia stream type storage property. These properties can be managed by the following storage scheme.

1. distributed virtual storage. According to the logical relation of the frame and the frame structure, the large data stream is distributed in the virtual space. Distributed memory structure for frame combination.

2. data frame storage properties of the stream storage. According to the distortion of each multimedia stream in the virtual space, the frame structure of the large data stream is designed based on the distorted response information of large data.

3. virtual space twisted interface fusion storage. From the heterogeneous attributes of multiple multimedia streams, the combination of the large data frames of each multimedia is perceived in the distorted interface. For these properties, the server 
control center is saved to the virtual space in accordance with the different distortions.

4. multidimensional storage of twisted frame in virtual space. From the entity service group statistics of the large data size, the flow of communication bandwidth, virtual twisted large data frame sequence, the establishment of multi-dimensional frame combination storage architecture.

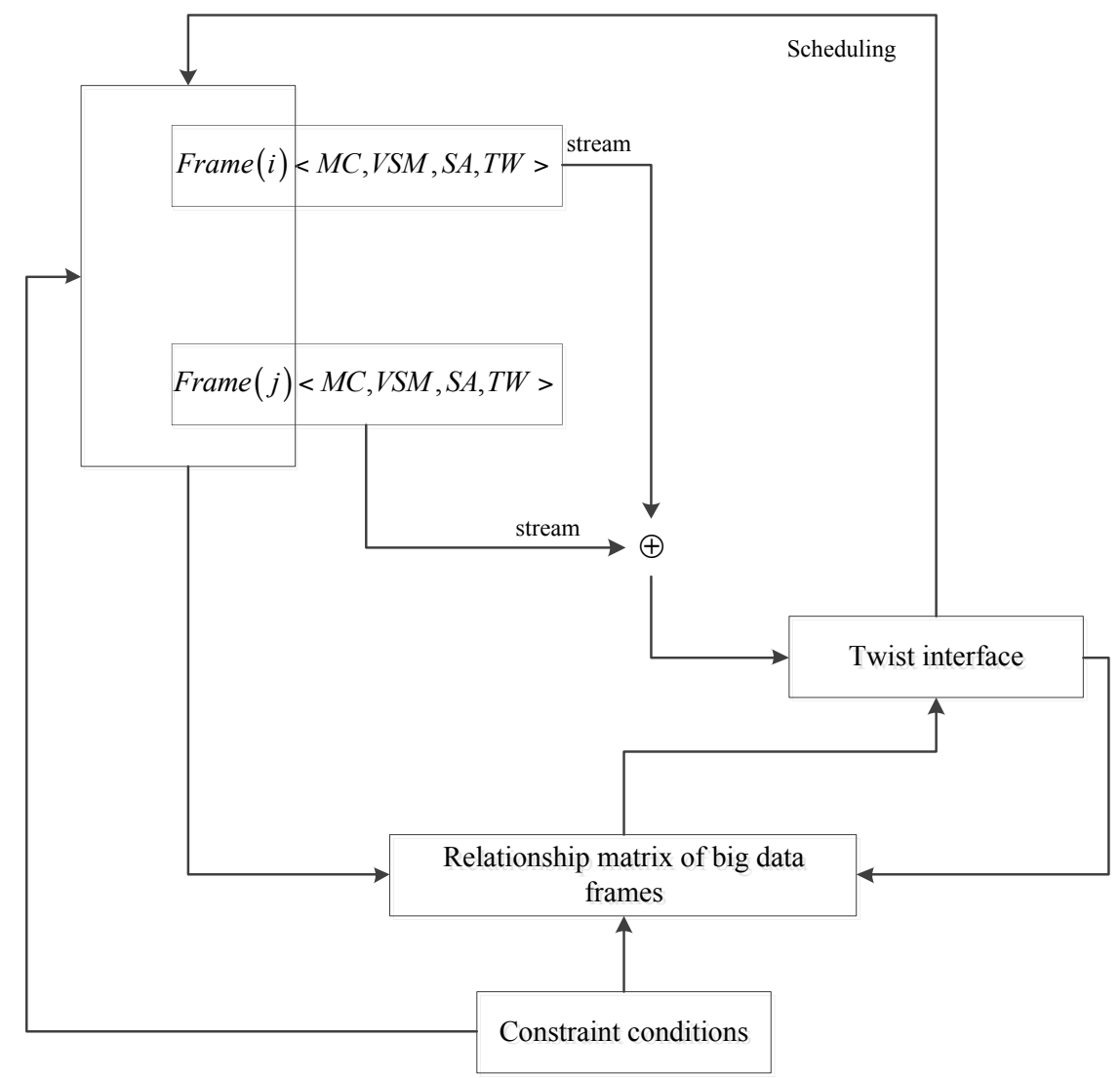

Fig. 3. Segmentation of virtual space

\section{Performance Analysis of Big Data Storage Mechanism}

For testing the performance of the proposed Multimedia big data frame combination storage strategy based on virtual space distortion denoted as MBCSVSD, we simulate multiple multimedia streams with concurrent communication and can respond to multiple users of multimedia requests in real time. From real time, storage efficiency, buffer duty cycle and channel idle rate and other aspects of verification and comparison of the proposed scheme and the physical server data 
Short Paper-Multimedia Big Data Frame Combination Storage Strategy Based on Virtual Space Distortion

storage scheme is recorded as PSDS. The hardware and software of the test system are described in Table 1.

Table 1. Setting of experiment

\begin{tabular}{|l|c|l|c|}
\hline \multicolumn{1}{|c|}{ Parameter } & Value & \multicolumn{1}{c|}{ Parameter } & Value \\
\hline Number of servers & 10 & Memory & $8 \mathrm{~GB}$ \\
\hline Number of CPU in server & 2 & Hard disk speed & $7200 \mathrm{rpm}$ \\
\hline main frequency of CPU & $2.5 \mathrm{GHz}$ & Communication protocol & IEEE $802.11 \mathrm{n}$ \\
\hline second level of CPU & $1 \mathrm{MB}$ & Wireless bandwidth & $100 \mathrm{MB} / \mathrm{s}$ \\
\hline Operating system & CentOS 5.0 & Programming language & Python \\
\hline
\end{tabular}

The virtual space of the unit distance is divided into $1200 * 1500$ cells. Random distribution of multimedia streaming in virtual environment. Experimental statistical rules are as follows:

5. in the real time testing, the simulation of the 20 multimedia stream concurrent processing and communication. Test and record the virtual space of each cell were randomly generated $2,3,1,4,5$ twisted time to complete all the big data storage tasks required, the results shown in figure 4.

6. in the storage efficiency test, the ratio of the frame to frame rate increases from $1 \%$ to $10 \%$. Step size $1 \%$. The utilization rate of the statistical server storage space through the virtual space distortion sensing interface is shown in Figure 5.

7. buffer duty cycle test, 10 server. Each of these servers run 5 parallel multimedia streams. Large scale multimedia data from $100 \mathrm{MB}$ to $1 \mathrm{~GB}$ gradually increased, the step size is $100 \mathrm{MB}$. Respectively, the ability of the system to empty the buffer queue of these large data buffer queue and channel utilization, the results shown in Figure 6 and 7.

From the point of view of Figure 4, whether it is the storage mechanism of the virtual space or the storage scheme of the physical server, the delay increases with the number of requests. However, the proposed scheme has a better parallel processing performance, and the delay of the rising trend is slow. With the increase of the number of requests, the delay of the proposed scheme tends to be more and more slowly. The main reason is that with the increase of the number of users, the storage system of virtual space processing time consuming and distorted perception interface processing time is almost negligible.

The test was repeated 5 times, and the average results were shown in figure 5 . The number of multimedia streams processed by each server is almost equal. The storage efficiency decreases with the increase of the frame rate. The storage efficiency of the two storage schemes is different. The proposed scheme is dominant. It is mainly because of the proposed scheme that the distortion of the entity data is converted to the distortion of the virtual space. The balanced processing of high quality video frame cannot be realized by the physical server storage scheme. 
Short Paper-Multimedia Big Data Frame Combination Storage Strategy Based on Virtual Space Distortion

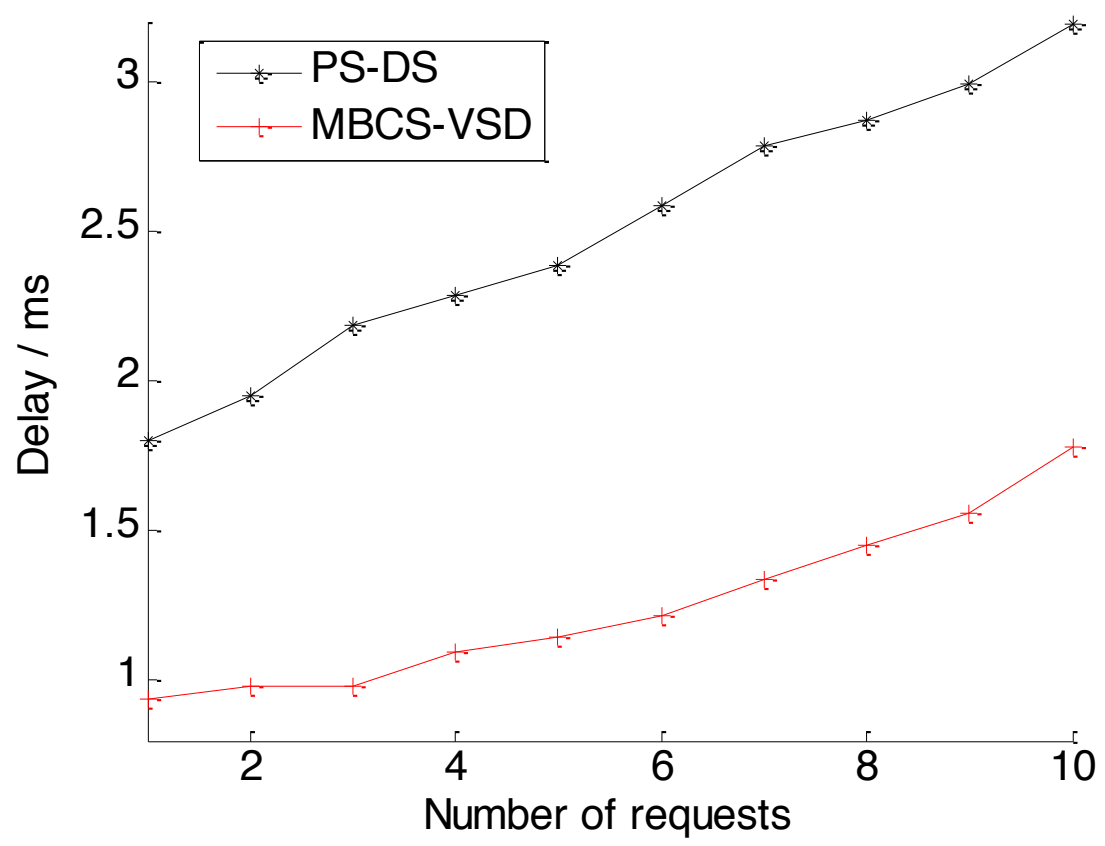

Fig. 4. Delay

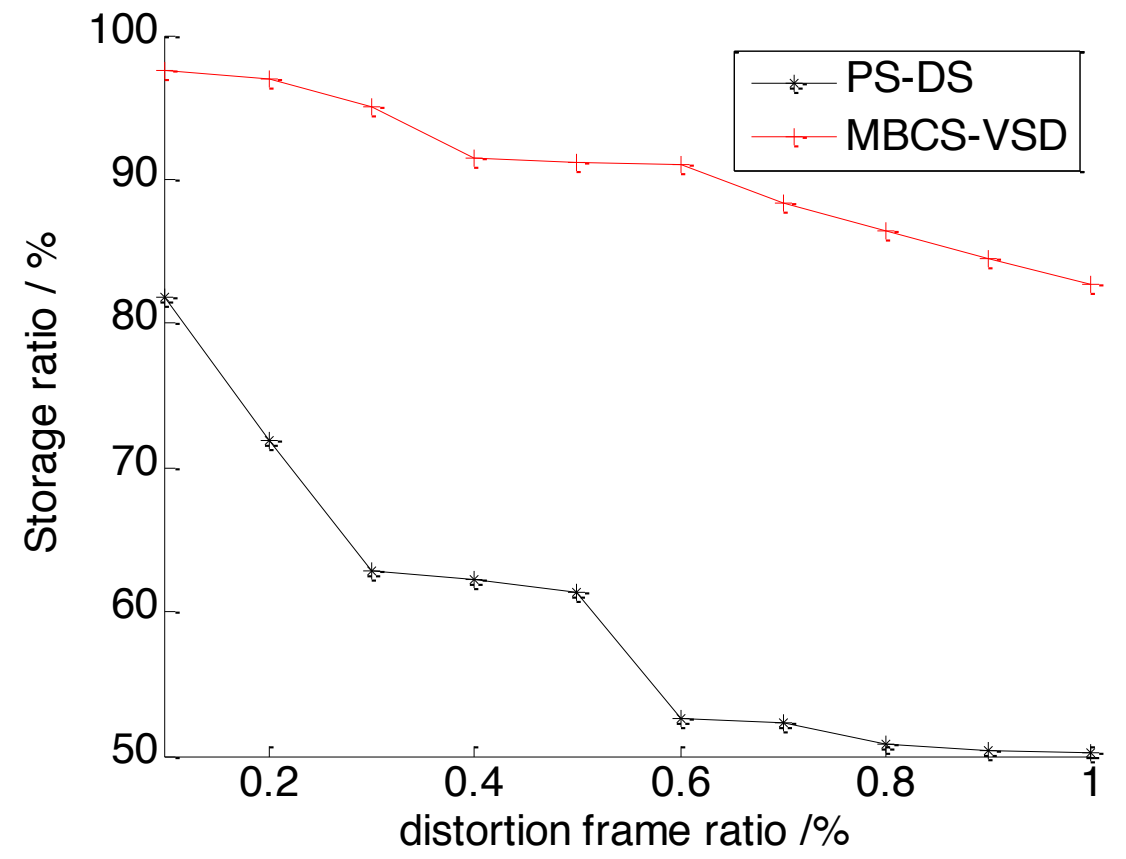

Fig. 5. Storage ratio 
Short Paper-Multimedia Big Data Frame Combination Storage Strategy Based on Virtual Space Distortion

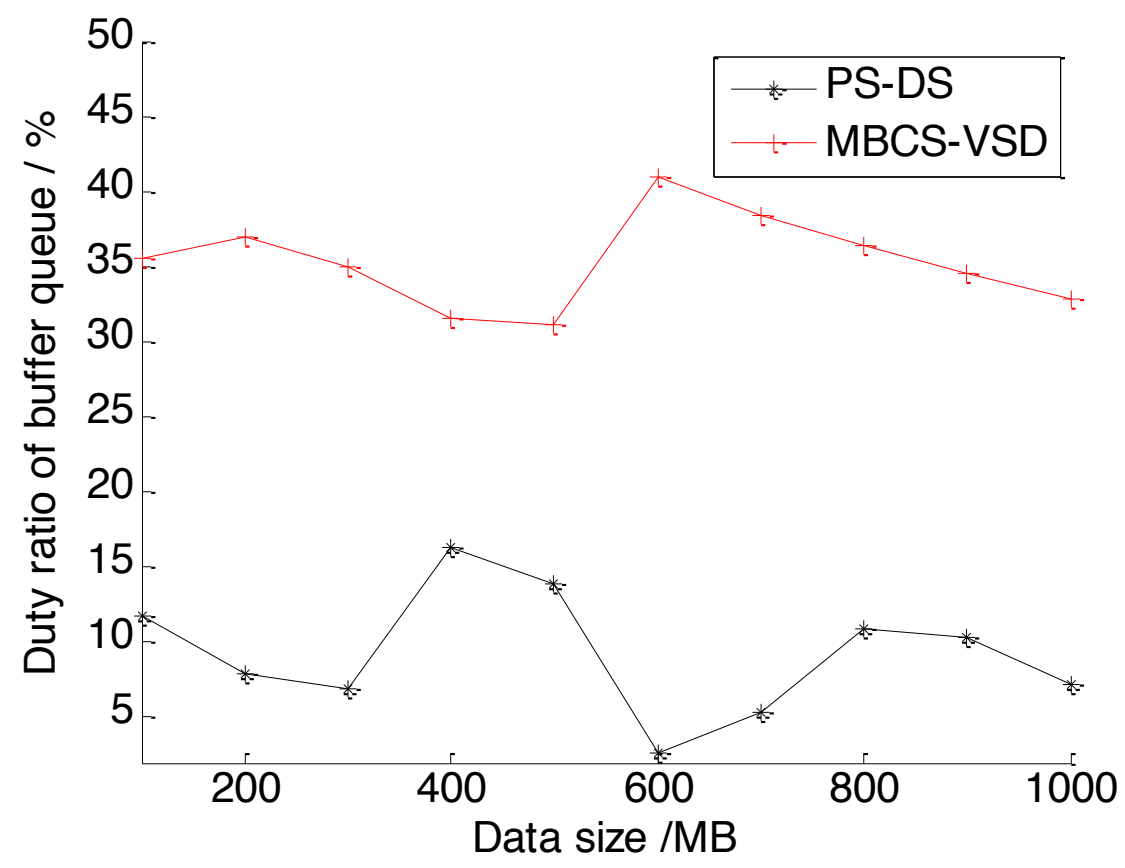

Fig. 6. Duty ratio of buffer queue

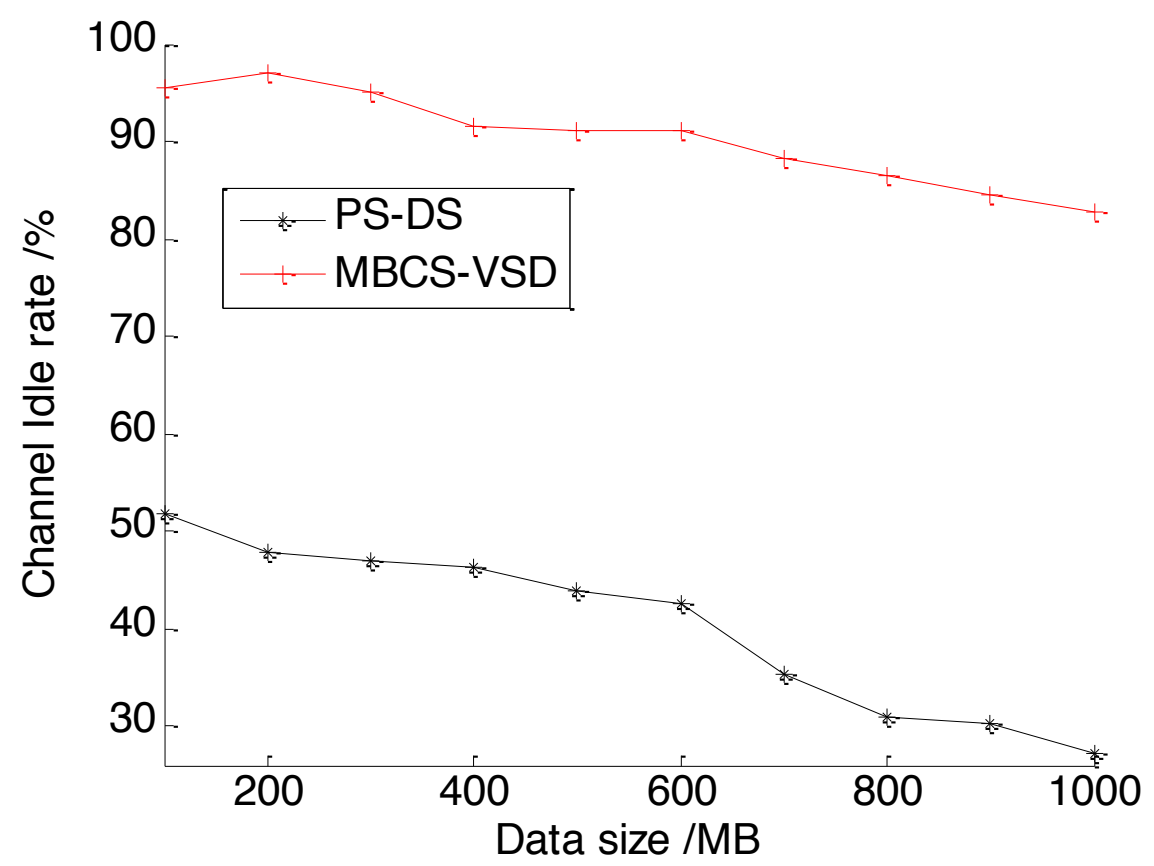

Fig. 7. Channel idle rate 
Through the comparison of figures 6 and 7 show that redundant frame storage management algorithm of virtual space distorted perception produces less than the physical server storage algorithm, you can quickly clean up the buffer queue and calls the wireless channel for the transmission of multimedia stream. This is mainly due to the storage management algorithm using virtual space warp perception, in order to meet the constraints of frame structure of large data storage demand and multimedia storage system, select the storage mechanism according to the actual situation of different. Distributed virtual storage, large data frame storage properties of the stream storage, virtual space distortion interface of the integration of storage, virtual space frame combination of multi-dimensional storage. From the entity service group statistics of the large data size, the flow of communication bandwidth, virtual twisted large data frame sequence, the establishment of multi-dimensional frame combination storage architecture.

\section{Conclusions}

In order to improve the reliability and the efficiency of large data storage in multimedia communication, a large data frame combined storage strategy based on virtual space is proposed. First, find the video frame high similarity and flow structure in different multimedia streams, through processing and server data flow within the communication state consistency, the establishment of virtual space warp analysis model, in order to reduce the difference of data mining multimedia stream weakening virtual space complexity and the. Secondly, the multimedia big data service is based on the heterogeneous data and distorted virtual space, which provides a description model for multimedia services. For large data multimedia services based on virtual space, multimedia services according to the data frame combination expression by distorting the logical drive, large data frame combination of storage space and virtual space integration as a whole. A combination of multimedia data frames is proposed. In the end, the experiment results of the simulation of the multimedia data show the real-time performance, the channel utilization, the storage efficiency and the large data processing capability of the proposed storage strategy.

\section{Acknowledgment}

This work is funded by the Public Welfare Technology Application Research Foundation of Zhejiang Province under grant No. 2015C31107 and Higher Education and Teaching Reform Foundation of Zhejiang Province under grant No.JG2015367.

\section{$7 \quad$ References}

[1] Wang H, Qian Y, Sharif H. "Multimedia communications over cognitive radio networks for smart grid applications", IEEE Wireless Communications, vol. 20 no. 18, 2013, p125132. https://doi.org/10.1109/MWC.2013.6590059 
[2] $\mathrm{Bu} \mathrm{S}$, Yu F R. "Green Cognitive Mobile Networks With Small Cells for Multimedia Communications in the Smart Grid Environment", IEEE Transactions on Vehicular Technology, vol. 63, no. 5, 2014, p2115-2126. https://doi.org/10.1109/TVT.2014.2313604

[3] Chang S, Chiao H, Hung Y. "Ideal Forward Error Correction Codes for High-Speed Rail Multimedia Communications", IEEE Transactions on Vehicular Technology, vol. 63, no. 8, 2014, p3517-3529. https://doi.org/10.1109/TVT.2014.2310897

[4] Li P, Guo S, Hu J. "Energy-Efficient Cooperative Communications for Multimedia Applications in Multi-Channel Wireless Networks", IEEE Transactions on Computers, vol. 64, no. 6, 2015, p1670-1679.

[5] Bhattacharya A, Ghosh R, Sinha K, et al. "Non-Contiguous Channel Allocation for Multimedia Communication in Cognitive Radio Networks", IEEE Transactions on Cognitive Communications \& Networking, vol. 1, no. 4, 2015, p420-434. https://doi.org/10.1109/TCCN.2016.2549033

[6] Yu Q Y, Meng W X, Yang M C, et al. "Virtual multi-beamforming for distributed satellite clusters in space information networks", IEEE Wireless Communications, vol. 23, no. 1, 2016, p95-101. https://doi.org/10.1109/MWC.2016.7422411

[7] Tian K, Wang J, Wu B, et al. "A Virtual Space Vector Modulation Technique for the Reduction of Common-Mode Voltages in Both Magnitude and Third-Order Component", IEEE Transactions on Power Electronics, vol. 31, no. 1, 2016, p839-848. https://doi.org/10.1109/TPEL.2015.2408812

[8] Singh D, Lee J W, Hussain S, et al. "Merge-and-Forward: A Cooperative Multimedia Transmissions Protocol using RaptorQ Codes", IET Communications, vol. 10, no. 15, 2016, p1884 - 1895. https://doi.org/10.1049/iet-com.2016.0146

[9] Yuan L, Li H, Wan Y. "A Novel UEP Fountain Coding Scheme for Scalable Multimedia Transmission", IEEE Transactions on Multimedia, vol. 18, no. 7, 2016, p1389-1400. https://doi.org/10.1109/TMM.2016.2557079

[10] Xing M, He J, Cai L. "Maximum-Utility Scheduling for Multimedia Transmission in Drive-Thru Internet", IEEE Transactions on Vehicular Technology, vol. 65, no. 4, 2015, p1-1.

[11] Moyne J, Samantaray J, Armacost M. "Big Data Capabilities Applied to Semiconductor Manufacturing Advanced Process Control", IEEE Transactions on Semiconductor Manufacturing, vol. 29, no. 4, 2016, p283 - 291. https://doi.org/10.1109/TSM.2016. 2574130

[12] Fegaras L. "Incremental Query Processing on Big Data Streams", IEEE Transactions on Knowledge and Data Engineering, vol. vol.28, no. 11, 2016, p2998 - 3012.

[13] Hernandez A F R, Garcia N Y G. "Distributed processing using cosine similarity for mapping Big Data in Hadoop", IEEE Latin America Transactions, vol.14, no. 6, 2016, p2857-2861. https://doi.org/10.1109/TLA.2016.7555265

\section{Author}

Jian Luo received the Master's degree from Wuhan University of Technology in 1996. He is an associated professor in the school of Digital Information at Zhejiang Technical Institute of Economics, Hangzhou Zhejiang, 310018 China. His research interests include data mining, grid computing and embedded system, etc. (jiansluo@yahoo.com) 